$(F=19.0, \mathrm{df}=2 / 27, \mathrm{p}>.05)$. The postshock approach latencies for the operated Ss were also shorter, and only one (5\%) of these Ss avoided the spout compared to five (42\%) of the control Ss. The Kruskal-Wallis one-way analysis of variance by ranks (Siegel, 1956) performed on postshock approach latencies for the three groups was statistically significant $(H=8.25, p<.05)$. A planned Mann-Whitney test on approach latencies was performed between Groups $\mathrm{HpL}$ and HpESB. No significant difference was found between the two operated groups $(z=.62)$.

Examination of electrode placements indicated that in at least one S (CA33-6 in Fig. 1), the electrode was stimulating the cortex dorsal to the hippocampus. This $\mathrm{S}$ accepted the fewest shocks and had the longest postshock approach latency in Group HpESB. Other comparisons between electrode locus and performance revealed that those placements which were most likely stimulating the hippocampus produced the greatest deficits in avoidance. There was similar agreement between the extent of damage in Group $\mathrm{HpL}$ and avoidance behavior. For example, the $S$ with the most hippocampal tissue remaining (some dorsal, most ventral) was the only operated $S$ that did not approach the water spout during the retention session.

The results indicate that changes in passive avoidance behavior produced by electrical stimulation are similar to those observed in hippocampally lesioned rats. The present findings are in accord with the view (Kasper, 1964) that constant stimulation of certain related limbic structures is capable of disrupting a circuit which mediates response inhibition. Differences in response inhibition may also have resulted from an increased drinking tendency on the part of experimental Ss (Kimble \& Coover, 1966). The contribution of consummatory factors might be assessed by removing ESB during the postshock retention test.

\section{REFERENCES}

ISAACSON, R. L., \& WICKELGREN, W. O Hippocampal ablation and passive avoidance. Science, 1962,133, 1104-1106.

KASPER, P. Attenuation of passive avoidance by continuous septal stimulation. Psychonomic Science, 1964, 1, 219-220.

KIMBLE, D. P., \& COOVER, G. D. Effects of hippocampal lesions on food and water consumption in rats. Psychonomic Science 1964, 4, 91-92.

SIEGEL, S. Nonparametric statistics for the behavioral sciences. New York: McGraw-Hill 1956.

NOTE

1. The authors would like to thank Dr. Rober M. Stutz for his critical reading of this paper.

\title{
Varying temporal relationship of an intruded aversive stimulus with time-out from responding ${ }^{1}$
}

A. G. SNAPPER and E. H. SHIMOFF, Franklin Delano Roosevelt V.A. Hospital, Montrose, N.Y. 10548, and W. N. SCHOENFELD, Queens College, Flushing, N.Y. 11367

Four groups of rats were trained on a random-interval schedule of positive reinforcement. During daily sessions successive 2-min exposures to the schedule were interrupted by 2-min time-outs during which the lever operandum was withdrawn from the chamber. Response-independent shock was superimposed in different portions of the behavior stream for different groups so that one group was shocked in the middle of the reinforced periods, one at the end of the reinforced components, one group in the middle, and one group at the end of the time-out components. Animals shocked during or at the end of the reinforced component were suppressed by lower intensity shocks than were rats shocked outside the periods of bar-pressing. The results are consistent with several different conceptual schemes.

Farmer \& Schoenfeld (1966a) have reported an experiment involving an "irreducibly primitive paradigm for exploring stimulus effects on steady-state responding." In that study, the position of an intruded stimulus was varied systematically within a 1-min fixed-interval schedule of positive reinforcement. The degree of control exerted by the stimulus was considerable, and was closely related to the time of its intrusion. An earlier study by Flanagan \& Webb (1964) had similar results and, in a second experiment, Farmer \& Schoenfeld (1966b) demonstrated that response-independent stimulus onset at different portions of a fixed-interval schedule also exerted control over responding. These studies demonstrate that the stimulus intrusion paradigm can resemble both that of conditioned reinforcement and of discriminative stimuli.
The present experiment extends the paradigm to the case where the intruded stimulus is "aversive" (electric shock). The stimulus intrusion was effected during times when the behavior stream was dominated by a positively reinforced prespecified response ( $R$, bar-pressing by rats), and during the times when nonspecified behavior other than bar-pressing (not-R) was in progress. In this case the paradigm can be seen to resemble the procedures of delay of punishment and of conditioned suppression. SUBJECTS

The Ss were 16 male albino rats, $150-200$ days old, maintained at $80 \%$ of their free-feeding weights at the start of the experiment by water deprivation. Food was always available in the home cages, while sufficient water to maintain the desired body weight was given immediately following the sessions.

\section{APPARATUS}

The experimental chambers were Scientific Prototype rat chambers (Model A100) fitted with retractable levers. A $2-\mathrm{kHz}$, 75-dB tone was presented through speakers mounted directly above the experimental chambers. The speakers and chambers were enclosed in sound-attenuating shells (Scientific Prototype Model 300). The reinforcement was $0.01 \mathrm{cc}$ of Alba skim milk, presented for $4 \mathrm{sec}$. Experimental contingencies and data recording were programmed on a digital computer (Snapper, Knapp, \& Kushner, 1967). The shock was $325 \mathrm{~V}$ ac, distributed to the grids by a grid scrambler described by Snapper (1966). PROCEDURE

The rats were randomly divided in to four groups of four rats each. After initial "shaping" of the bar-pressing response, they were exposed to the baseline schedule, which consisted each day of 302 -min periods comprising a 1 h session. In the first period and alternate periods following it (i.e., the third, fifth, seventh, etc.), the bar 


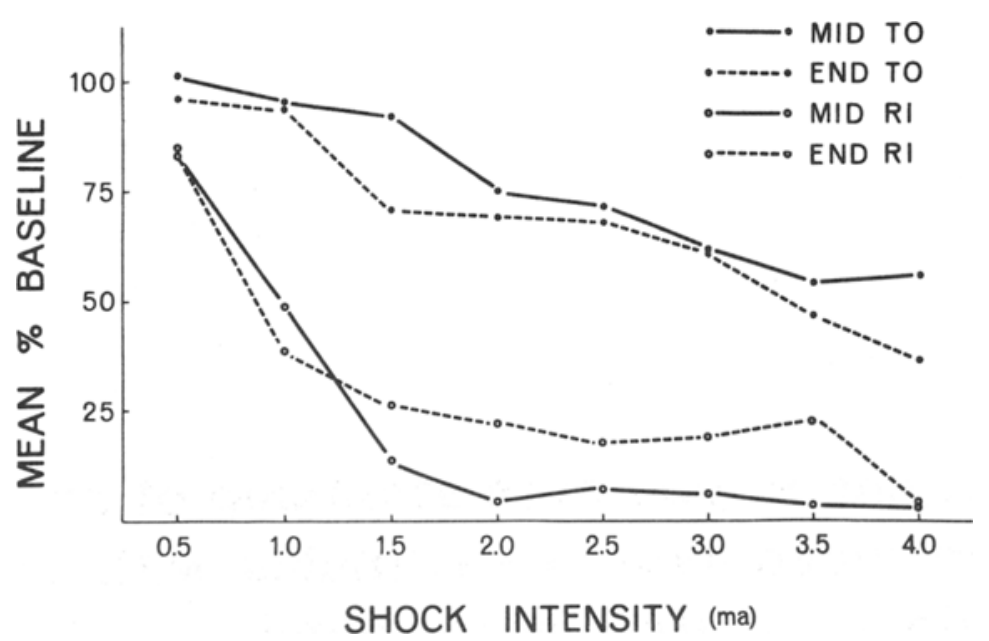

Fig. 1. Mean response rates for the four groups as a function of shock intensity. Each point represents the mean of four subjects over the three days at each intensity. was available, the tone was presented, and reinforcements were available on a variation of a 5-sec random-interval (RI, Farmer, 1963) schedule in which the interval was restarted at the end of the reinforcement so that the smallest possible interreinforcement time was $5 \mathrm{sec}$. Interpolated between these RI periods were time-out (TO) periods (for the second, fourth, sixth periods, etc.) during which the bar was withdrawn and the tone was removed. After response rates had stabilized, five shocks were presented in each session in a pseudorandom order, with the constraint that at least two periods (one RI and one TO) separate shock presentations. Response-independent shocks were presented either in the middle of an RI period (MRI group), at the end of an RI period (ERI), in the middle of a TO period (MTO), or at the end of a TO period (ETO), regardless of the behavior of the $S$. The

shock intensity was initially set at $0.5 \mathrm{~mA}$ and increased in $0.5-\mathrm{mA}$ steps every 3 days to a final value of $4.0 \mathrm{~mA}$.

RESULTS AND DISCUSSION

Mean response rates averaged over 3-day blocks were calculated as a percentage of the nonshocked baseline rate and are plotted in Fig. 1. Response suppression was greater in the two groups (MRI and ERI) in which shocks came during or immediately after RI periods than in the two groups in which shocks came during or following TO periods. In both cases, the disruptive effects of shock were greater as shock intensity was increased. Analysis of variance showed a significant effect of shock intensity and a significant difference between groups $(p<.01)$. The interaction variable was not significant $(p>.05)$, indicating that the effects of shock intensity were parallel for all groups. Further analysis indicated that

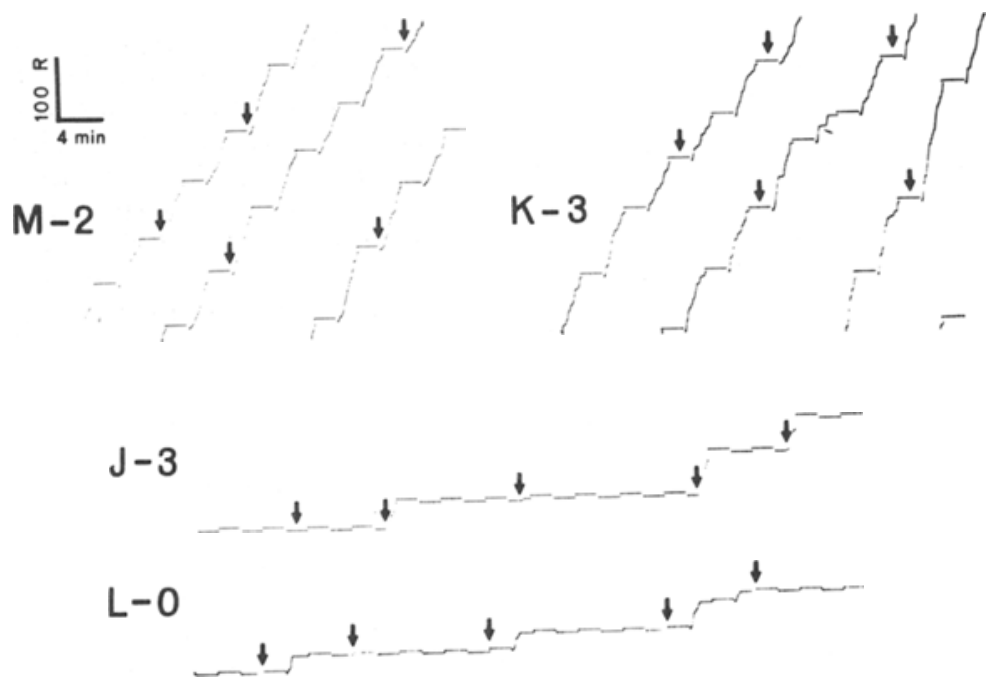

Fig. 2. Cumulative response curves for individual subjects at $4.0 \mathrm{~mA}$. The pen is deflected downward during RI periods. Arrows indicate shock presentation. Subject M-2 was shocked at the end of time-out, K-3 in the middle of time-out, J-3 in the middle of the RI segment and L-O was shocked at the end of RI. there were no differences between either ERI and MRI groups or the ETO and MTO groups $(\mathrm{p}>.05)$, but that the differences between the ETO and the MTO groups vs the ERI and the MRI groups was significant ( $<<.01$ ). Cumulative records (Fig. 2) show that the typical pattern of responding under high-intensity shock for the MRI group involved suppression at the start of the session, with responding immediately following shock, while responding in the ERI group was primarily in the $\mathrm{RI}$ period following shock presentation. Suppression in the ETO and MTO groups was approximately uniform throughout the session.

The greater level of suppression in the groups shocked during and at the end of reinforced responding over those punished in the TO is consistent both with the delay-of-punishment literature (Cohen, 1968) and with conditioned-emotionalresponse studies (Seligman, 1968). Moreover, Kelleher \& Morse (1961) have already noted the similarity between delay of punishment and conditioned suppression. It is clear that the intruded stimulus paradigm can take on the properties of classical paradigms when superimposed upon different portions of the behavior stream.

\section{REFERENCES}

COHEN, P. S. Punishment: The interactive effects of delay and intensity of shock. Journal of the Experimental Analysis of Behavior, 1968, 11, 789-799.

FARMER, J. Properties of behavior under random interval schedules of reinforcement. Journal of the Experimental Analysis of Behavior, 1963,6, 607-616.

FARMER, J., \& SCHOENFELD, W.N. The effects of a response-contingent stimulus introduced into a fixed-interval schedule at varying temporal placement. Psychonomic Science, $1966 \mathrm{a}, 6,15-16$.

FARMER, J., \& SCHOENFELD, W. N. Varying temporal placement of an added stimulus in a fixed-interval scheduk. Journal of the Experimental Analysis of Behavior, $1966 \mathrm{~b}, 9$, 369-375.

FlaNAGAN, B., \& WEBB, W. B. Disinhibition and ex ternal inhibition of fixed interval operant conditioning. Psychonomic Science, 1964, 1, 123-124.

KELLEHER, R. T., \& MORSE, W. H. Escape behavior and punished behavior. Federation Proceedings, 1964, 23, 808-817.

SELIGMAN, M. E. P. Chronic fear produced by unpredictable shock. Journal of Comparative \& Physiological Psychology, 1968, 66, 402-411

SNAPPER, A. G. A relay-transistor sequential grid scrambler. Journal of the Experimental Analysis of Behavior, 1966, 9, 173-176.

SNAPPER, A. G., KNAPP, J., \& KUSHNER, H. Online programming: I. Paper presented at the meeting of the American Psychological Association, Washington, D.C., September 1967. NOTE

1. This research was supported by Grant MH-13049 from the National Institute of Mental Health, USPHS, to William N. Schoenfeld, Queens College, New York, and by the Veterans Administration. Reprints may be obtained from the Psychology Research Laboratory, FDR VA Hospital, Montrose, New York 10548. 